\title{
In Vivo Metabolism of Proapolipoprotein A-I in Tangier Disease
}

Dubo Bojanovski, Richard E. Gregg, Loren A. Zech, Martha S. Meng, Cheri Bishop, Rosemary Ronan, and H. Bryan Brewer, Jr. Molecular Disease Branch, National Heart, Lung, and Blood Institute, National Institutes of Health, Bethesda, Maryland 20892

\begin{abstract}
Tangier disease is a rare familial disorder characterized by extremely low levels of apolipoprotein A-I (apoA-I) and high density lipoproteins (HDL). In normal subjects, proapoA-I is secreted into plasma and converted to mature apoA-I by the cleavage of the amino-terminal six amino acids with the major isoprotein in plasma being mature apoA-I. In contrast, in Tangier disease there is a marked relative increase of proapoA-I as compared with mature apoA-I. ProapoA-I and mature apoA-I were isolated from normal and Tangier disease subjects, radiolabeled, and autologous apoA-I isoproteins injected into normal and Tangier subjects. The in vivo catabolism and conversion of proapoA-I and mature apoA-I in normal and Tangier disease subjects were quantitated. A comparison of the rate of catabolism of apoA-I isoproteins from plasma revealed a significantly faster rate of catabolism of both isoproteins of apoA-I in Tangier subjects when compared with normal subjects. The fractional conversion rate of proapoA-I to mature apoA-I was $3.9 d^{-1}$ in normal subjects and $3.6 d^{-1}$ in Tangier subjects. The results indicate that $(a)$ apoA-I enters plasma as the pro isoprotein in both normal and Tangier subjects, (b) Tangier disease subjects have a normal fractional rate of conversion of proapoA-I to mature apoA-I, (c) proapoA-I is catabolized at the same rate as mature apoA-I in Tangier subjects, and $(d)$ Tangier subjects catabolize both pro and mature apoA-I at a much greater rate than do normal subjects. Therefore, the relative increase in proapoA-I in Tangier disease is due to a marked decrease in mature apoA-I resulting from rapid catabolism of both pro- and mature apoA-I and not to defective conversion of proapoA-I to mature apoA-I.
\end{abstract}

\section{Introduction}

Tangier disease is a rare familial syndrome characterized by hypocholesterolemia, moderate hypertriglyceridemia, abnormal chylomicron remnants, and plasma concentrations of HDL-cholesterol as well as apoA-I and apoA-II of $\sim 1-5 \%$ of normal values (1). Clinically, these patients have enlarged orange tonsils, hepatosplenomegaly, lymphadenopathy, intermittent peripheral neuropathy, and there is a possible increased incidence of premature atherosclerotic vascular disease (2). Initial metabolic studies from our laboratory established that Tangier subjects were able to synthesize apoA-I at a normal rate and the deficiency of plasma apoA-I was due to accelerated catabolism $(3,4)$.

Address reprint requests to Dr. Gregg, Building 10, Room 7N117, NIH, Bethesda, MD 20892.

Portions of this manuscript were previously published in abstract form in 1984. (Clin. Res. 32:390a).

Received for publication 21 January 1987 and in revised form 27 July 1987.

The Journal of Clinical Investigation, Inc.

Volume 80, December 1987, 1742-1747
The precursor of human apoA-I, preproapoA-I, is a 267 amino acid protein containing 24 additional amino acids attached to the amino-terminal end of the mature apoA-I. 18 amino acids are contained within the prepeptide followed by a six amino acid propeptide. PreproapoA-I undergoes cotranslational proteolytic cleavage to proapoA-I, which is secreted into human plasma or lymph (apoA- $\mathrm{I}_{+1,+2}$ isoforms) still containing the six amino acid propeptide attached to the aminoterminus of mature apoA-I $(5,6)$. In normal subjects proapoA-I is converted in plasma to mature apoA-I (apoA- $\mathrm{I}_{0,-1,-2}$ isoforms) by a specific calcium requiring peptidase (7-9). ApoA- $\mathrm{I}_{+2}$ and apoA- $\mathrm{I}_{\mathrm{o}}$ are the major pro and mature isoforms, respectively. In addition, there is a very slow loss of 1 or 2 positive changes from proapoA-I and mature apoA- $I_{o}$ without proteolysis to generate the respective minor isoforms, apoA- $\mathrm{I}_{+1}$ and apoA-I $\mathrm{I}_{-1,-2}(9)$. Human proapoA-I is a relatively minor isoprotein in plasma, comprising $\sim 5 \%$ of the total circulating apoA-I. Patients with Tangier disease have a marked increase in plasma of proapoA-I relative to mature apoA-I (10). This observation led to the speculation that the biochemical abnormality in Tangier disease is a defect in the conversion of proapoA-I to mature apoA-I, resulting in rapid catabolism of proapoA-I and low plasma apoA-I levels $(5,10,11)$. We have subsequently demonstrated that Tangier plasma can convert proapoA-I to mature apoA-I at a normal rate in in vitro experiments (8); the reason, however, for the relative increase in proapoA-I in Tangier disease subjects remained unknown. Therefore, in order to elucidate the kinetic etiology of the altered apoA-I isoform pattern in Tangier disease, we have performed metabolic studies with purified proapoA-I and mature apoA-I in normal subjects and patients with Tangier disease.

\section{Methods}

Study subjects. The study subjects included two normal volunteers and two homozygous Tangier disease subjects including a member (subject 2) from the initial family described with this disease (12). The results for the normal subjects have been previously published (9). All of the subjects had normal hepatic, renal, and endocrine function and were in good health at the time of the study. The study protocol was approved by the Institute Review Board of the National Heart, Lung, and Blood Institute and informed consent was obtained from each subject. The studies were performed while the subjects were inpatients on the metabolic ward of the Clinical Center of the National Institutes of Health. Starting $10 \mathrm{~d}$ before the study, the subjects began an iso-weight diet containing $16 \%$ protein, $42 \%$ fat, $42 \%$ fat (polyunsaturated/saturated fat ratio of 0.2 ), and $200 \mathrm{mg}$ cholesterol $/ 1,000 \mathrm{kcal}$. Subjects were given potassium iodide $(0.9 \mathrm{~g} / \mathrm{d})$ in divided doses beginning $1 \mathrm{~d}$ before the injection of the radiolabeled apolipoproteins and continuing for the duration of the study to inhibit thyroid uptake of radioactivity.

Quantification and isolation of plasma lipids, lipoproteins, and apolipoproteins. Blood was collected after a 12-h fast in EDTA $(0.1$ $\mathrm{mg} / \mathrm{ml}$ ) and the plasma separated by centrifugation. Thoracic duct lymph was obtained from patients undergoing thoracic duct drainage and lymph chylomicrons were obtained by centrifugation at $d=1.006$ $\mathrm{g} / \mathrm{ml}$ for $0.5 \mathrm{~h}$ at $27,000 \mathrm{rpm}$ in a 60 -Ti rotor in an ultracentrifuge (both 
from Beckman Instruments, Inc., Palo Alto, CA). Lipoproteins from Tangier patients were obtained by ultracentrifugation of plasma $\left(53,000 \mathrm{rpm}, 4^{\circ} \mathrm{C}\right)$, at $1.21 \mathrm{~g} / \mathrm{ml}$ in a $60 \mathrm{Ti}$ rotor for $18 \mathrm{~h}$. Normal HDL were isolated by ultracentrifugation of plasma at $d 1.063-1.21 \mathrm{~g} / \mathrm{ml}$ using $\mathrm{KBr}$ for density adjustment as previously described (13). The protein content was determined by the method of Lowry et al. (14). Plasma lipid and lipoprotein cholesterol values were quantitated as previously described (9). Apolipoproteins were quantitated by radial immunodiffusion (15). The apolipoprotein A-I isoproteins were isolated as previously described $(9,16,17)$. Briefly normal proapoA-I (apoA- $\mathrm{I}_{+2}$ isoform) and mature apoA-I (apoA- $\mathrm{I}_{0}$ isoform) were isolated from lymph lipoproteins $(d<1.006 \mathrm{~g} / \mathrm{ml})$ and $\mathrm{HDL}$, respectively, by preparative isoelectrofocusing. Total apoA-I $\mathrm{I}_{\text {Tangier }}$ was purified from delipidated lipoproteins $(d<1.21 \mathrm{~g} / \mathrm{ml})$ by gel permeation chromatography and proapoA- $\mathrm{I}_{\text {Tangier }}$ as well as mature apoA- $\mathrm{I}_{\text {Tangier }}$ isolated by preparative isoelectrofocusing (17).

Radioiodination of apolipoprotein A-I isoproteins. Purified normal and Tangier isoproteins $(50-100 \mu \mathrm{g})$ were dissolved in $25-50 \mu \mathrm{l}$ of $6 \mathrm{M}$ guanidine- $\mathrm{HCl}, 1 \mathrm{M}$ glycine (pH 8.50) and iodinated with ${ }^{125} \mathrm{I}$ or ${ }^{131} \mathrm{I}$ (New England Nuclear, Boston, MA) by the iodine monochloride method $(18,19)$. Less than $1 \mathrm{~mol}$ of iodine was incorporated per $2 \mathrm{~mol}$ of isoprotein with the efficiency of iodination being assessed by precipitation of protein with $20 \%$ trichloroacetic acid.

Recombination of radiolabeled apoA-I isoproteins with lipoproteins. Autologous radiolabeled apoA-I isoproteins were incubated with $35 \mathrm{ml}$ of normal or Tangier plasma containing EDTA for $60 \mathrm{~min}$ at room temperature, the density adjusted to $1.21 \mathrm{~g} / \mathrm{ml}$ using solid $\mathrm{KBr}$, and the samples centrifuged. Tangier plasma containing radiolabeled Tangier apoA-I isoproteins was centrifuged in a $60 \mathrm{Ti}$ rotor at 53,000 $\mathrm{rpm}, 4^{\circ} \mathrm{C}$, for $18 \mathrm{~h}$, and normal plasma containing radiolabeled apoA-I isoproteins was centrifuged in a $60 \mathrm{Ti}$ rotor at $59,000 \mathrm{rpm}, 4^{\circ} \mathrm{C}$, for 24 $\mathrm{h}$. The $1.21 \mathrm{~g} / \mathrm{ml}$ supernate lipoproteins were isolated by tube slicing and the unbound iodide removed by dialysis against $150 \mathrm{mM} \mathrm{NaCl}$, $0.01 \%$ EDTA, and $0.1 \mathrm{M}$ Tris- $\mathrm{HCl}(\mathrm{pH} 7.4)$. All preparations were filtered ( 0.22 micron filters; Millipore Corp., Bedford, MA) and tested for pyrogenicity, and sterility. Radiolabeled apoA-I reassociated with lipoproteins were analyzed by analytical isoelectrofocusing with unlabeled apoA-I standards to ascertain the electrophoretic position of the apoA-I isoforms (9).

Electrophoretic methods. Analytical two-dimensional gel electrophoresis was conducted as previously described (20). Isoelectrofocusing was performed at $250 \mathrm{~V}$ for $26 \mathrm{~h}$ with $3 \%$ ampholines ( $\mathrm{pH} \mathrm{5.0-6.0)}$ followed by $\mathrm{NaDodSO}_{4}$ slab gel electrophoresis (15\% acrylamide, $0.085 \%$ bisacrylamide). The relative proportion of the different isoforms of apoA-I on the two-dimensional gel was quantitated as previously reported (9) by densitometric scanning of the gel.

Preparative isoelectrofocusing was conducted with $300-600 \mu \mathrm{g}$ of protein per well ( 5 well gels) on $3 \times 100 \mathrm{~mm}$ slab gels, using either 1.21 $\mathrm{g} / \mathrm{ml}$ supernate apolipoproteins (Tangier plasma), chylomicron apolipoproteins (lymph), or HDL apolipoproteins (normal plasma) (17, 21 ). Lyophilized apolipoprotein samples were dissolved in aqueous 10 $M$ urea and fractionated by isoelectrofocusing on a dual slab cell (BioRad 220; Bio-Rad, Richmond, CA) with a 5.0-6.0 pH gradient (Serva Ampholines; Serva AB, Heidelberg, FRG) using gels containing 7.5\% acrylamide and $0.2 \%$ bisacrylamide. The upper tray buffer was $0.02 \mathrm{M}$ $\mathrm{NaOH}$ and the lower buffer $0.01 \mathrm{M} \mathrm{H}_{3} \mathrm{PO}_{4}$. The gels were electrophoresed for $18 \mathrm{~h}$ at $250 \mathrm{~V}$, the isoprotein bands visualized from a portion of the gel with Coomassie Blue G-25, and the isoprotein bands cut from the unstained gel, eluted, and concentrated as previously described (21)

Metabolic studies. Two normal subjects were injected simultaneously with $100 \mu \mathrm{Ci}$ of normal ${ }^{125} \mathrm{I}$ proapoA-I and $25 \mu \mathrm{Ci}$ of normal ${ }^{131}$ I mature apoA-I. Similarly, $100 \mu \mathrm{Ci}$ of ${ }^{125} \mathrm{I}$ proapoA-I $\mathrm{I}_{\text {Tangier }}$ and 25 $\mu \mathrm{Ci}$ of ${ }^{131} \mathrm{I}$ mature apoA-I ${ }_{\text {Tangier }}$ were injected in two homozygous Tangier disease subjects. The studies were performed for $14 \mathrm{~d}$ in normal subjects, and for 2 and $3 \mathrm{~d}$ in the Tangier patients. In normal individuals, blood samples were obtained in $0.1 \%$ EDTA just before injection of radiolabeled apoA-I, and after injection at $10 \mathrm{~min}, 1 \mathrm{~h}, 3 \mathrm{~h}, 6 \mathrm{~h}, 12 \mathrm{~h}$,
$24 \mathrm{~h}, 36 \mathrm{~h}, \mathrm{~d} 2, \mathrm{~d} 3, \mathrm{~d} 5, \mathrm{~d} 7, \mathrm{~d} 9, \mathrm{~d} 11$, and d 14. In Tangier patients blood samples were obtained prior to the injection, and at $10 \mathrm{~min}, 1 \mathrm{~h}$, $2 \mathrm{~h}, 4 \mathrm{~h}, 6 \mathrm{~h}, 12 \mathrm{~h}, 18 \mathrm{~h}, 24 \mathrm{~h}, 36 \mathrm{~h}, 48 \mathrm{~h}$, and $72 \mathrm{~h}$ after injection of radiolabeled lipoproteins. The radioactivities in plasma, normal HDL $(d=1.063-1.21 \mathrm{~g} / \mathrm{m})$, and the Tangier $1.21 \mathrm{~g} / \mathrm{ml}$ supernate lipoproteins were quantified in an autogamma counter (Packard Model 560; Packard Instrument Co., Inc., Downers Grove, IL). Desalted aliquots of $\mathrm{HDL}$ or $1.21 \mathrm{~g} / \mathrm{ml}$ supernate lipoproteins were lyophilized, delipidated with chloroform methanol $(2: 1)$, and the pellets dried under a stream of nitrogen. The protein was resolubilized in $10 \mathrm{M}$ urea and ampholines, and the apoA-I isoforms separated by preparative isoelectrofocusing (9). The gel segments corresponding to the individual apoA-I isoforms (apoA-I +2 to apoA-I -2 ) were cut and the radioactivity quantitated to determine the relative distribution of radioactivity among the apoA-I isoproteins. The sum of the radioactivity recovered in the five apoA-I isoforms was $75-85 \%$ of the initial total counts at each time point. The plasma residence times of the apoA-I isoproteins were determined by computer assisted multiexponential curve fitting using the SAAM27 computer program. The apoA-I production rates were calculated from the following formula: production rate $=($ apoA-I concentration $\times$ plasma volume $) /($ weight $\times$ residence time).

\section{Results}

Characterization of plasma lipoproteins and apolipoproteins. Plasma lipid and lipoprotein cholesterol concentrations of all study subjects are shown in Table I. As has been previously noted, plasma total cholesterol, HDL cholesterol, and LDL cholesterol values were lower in Tangier homozygotes than in normal subjects while VLDL cholesterol as well as plasma triglyceride values were higher in Tangier homozygotes than in normal subjects.

The plasma apoA-I isoforms from a normal subject and Tangier patient are illustrated in Fig. 1. In normal subjects the predominant apoA-I isoforms in plasma are the mature apoA$\mathrm{I}$ isoproteins (apoA- $\mathrm{I}_{0,-1}$ isoforms). ProapoA-I migrates in the apoA-I $I_{+1}$ and apoA-I $I_{+2}$ isoform positions and is only a relatively minor isoprotein in normal plasma; however, it is markedly increased in thoracic duct lymph after fat feeding (22). The isoform pattern of apoA-I $\mathrm{I}_{\text {Tangier }}$ contains approximately equal quantities of pro and mature apoA-I $\mathrm{I}_{\text {Tangier }}$. Purified normal, as well as Tangier, pro- and mature isoproteins migrated as single electrophoretic bands in $\mathrm{NaDodSO}_{4}$ electrophoresis and polyacrylamide electrophoresis at $\mathrm{pH} 8.9$ as previously described (17). In addition to the electrophoretic evaluation, the purity and type of apoA-I isoprotein was also established by Edman amino terminal analysis of the isolated isoproteins (17).

Recombination of radiolabeled apoA-I isoproteins with lipoproteins. Each radiolabeled apoA-I isoprotein was recombined with plasma lipoproteins before injection, and the free apoA-I separated from lipoprotein bound apoA-I by ultracentrifugation at $1.21 \mathrm{~g} / \mathrm{ml}$. For the normal proapoA-I and mature apoA-I, $82 \%$ and $84 \%$ recombined with plasma lipoproteins, respectively (mean of 2 values each), while $73 \%$ of proapoA$I_{\text {Tangier }}$ and $83 \%$ of mature apoA-I $I_{\text {Tangier }}$ recombined with plasma lipoproteins.

Metabolism of proapoA-I and mature apoA-I in normal and Tangier disease subjects. The plasma decay curves of radioiodinated normal proapoA-I and mature apoA-I in a normal subject are illustrated in Fig. 2. The figure depicts the whole plasma decay curves without fractionation into lipoproteins or apolipoprotein isoforms. From this it is apparent that the overall fractional catabolic rate of proapoA-I and its meta- 


\begin{tabular}{|c|c|c|c|c|c|c|c|c|c|}
\hline \multirow[b]{2}{*}{ Subjects } & \multirow[b]{2}{*}{ Age } & \multirow[b]{2}{*}{ Sex } & \multirow[b]{2}{*}{ Height } & \multirow[b]{2}{*}{ Weight } & \multirow{2}{*}{$\begin{array}{l}\text { Plasma } \\
\text { triglyceride }\end{array}$} & \multicolumn{4}{|c|}{ Cholesterol } \\
\hline & & & & & & Plasma & VLDL & LDL & HDL \\
\hline & $y r$ & & $\mathrm{~cm}$ & $\mathrm{~kg}$ & $m g / d l$ & $m g / d l$ & $m g / d l$ & $m g / d l$ & $m g / d l$ \\
\hline \multicolumn{10}{|l|}{ Normal } \\
\hline 1 & 23 & $\mathrm{~F}$ & 167 & 59 & 59 & 182 & 8 & 88 & 86 \\
\hline 2 & 20 & $\mathbf{M}$ & 180 & 71 & 69 & 232 & 10 & 146 & 76 \\
\hline \multicolumn{10}{|c|}{ Tangier disease } \\
\hline 1 & 62 & $\mathbf{M}$ & 178 & 76 & 143 & 59 & 16 & 39 & 4 \\
\hline 2 & 30 & $\mathrm{~F}$ & 175 & 76 & 384 & 55 & 25 & 28 & 2 \\
\hline
\end{tabular}

bolic product, mature apoA-I, is the same as mature apoA-I in normal subjects. The plasma decay curves of proapoA-I and mature apoA-I are very similar because normal subjects rapidly convert virtually all of the proapoA-I to mature apoA-I, and the mature apoA-I derived from proapoA-I is catabolized at the same rate as the radiolabeled mature apoA-I.

In order to quantitate the catabolism of both proapoA$\mathrm{I}_{\text {Tangier }}$ and mature apoA-I $\mathrm{I}_{\text {Tangier }}$, and the conversion of proapoA-I to mature apoA-I in Tangier disease subjects, the respective isoproteins of apoA-I were isolated from Tangier disease subjects, radioiodinated, reassociated with lipoproteins from the Tangier disease subjects, and injected into these subjects. Fig. 3 depicts the plasma decay curves of proapoA-I $\mathrm{I}_{\text {Tangier }}$ and mature apoA- $\mathrm{I}_{\text {Tangier }}$ injected into a Tangier disease subject. The decay curves are very similar indicating that both proapoA-I $\mathrm{I}_{\text {Tangier }}$ and its metabolic product, mature apoA$\mathbf{I}_{\text {Tangier }}$, are both catabolized from plasma at a similar fractional catabolic rate. If this was not the case, these two curves would not be virtually superimposable. Compared to apoA-I in normal subjects, both proapoA-I and mature apoA-I in Tangier disease subjects were catabolized at a much greater rate than the corresponding apoA-I isoproteins in normal subjects.

The kinetics of conversion of proapoA-I $\mathrm{I}_{\text {Tangier }}$ to mature apoA- $\mathrm{I}_{\text {Tangier }}$ for one of the Tangier disease subjects studied is illustrated in Fig. 4. ProapoA- $\mathrm{I}_{\text {Tangier }}$ and mature apoA-I $\mathrm{I}_{\text {Tangier }}$ were injected into this subject with Tangier disease, the $d$ $<1.21 \mathrm{~g} / \mathrm{ml}$ lipoproteins isolated from plasma at various time intervals, the apoA-I isoproteins isolated by isoelectrofocusing gel electrophoresis, and the quantity of radioactivity in each isoform band quantitated in a gamma counter. The proapoA-I isoprotein was catabolized at an extremely rapid rate with both direct catabolism from plasma and conversion to mature apoA-I. Mature apoA-I $\mathrm{I}_{\text {Tangier }}$ derived from proapoA-I $\mathrm{I}_{\text {Tangier }}$ was catabolized at the same rate as the injected radiolabeled mature apoA-I $\mathrm{I}_{\text {Tangier }}$. These results are qualitatively very similar to the results in normal subjects but quantitatively the fractional catabolic rates of both proapoA-I and mature apoA-I are much faster in the Tangier disease subjects.

The kinetics of apoA-I synthesis, conversion, and catabolism in normal and Tangier disease subjects were quantitated, and the results are summarized in Table II. ApoA-I is secreted into plasma as the proapoA-I isoprotein with little or no direct catabolism of proapoA-I in normal plasma, and it is converted to mature apoA-I with a $t_{1 / 2}$ of conversion of $\sim 4 \mathrm{~h}$. In the Tangier disease subjects, there is extremely rapid direct catabolism of both proapoA-I and mature apoA-I with a plasma residence time $<5 \%$ of apoA-I in normal subjects. In contrast, the $t_{1 / 2}$ of conversion of proapoA-I to mature apoA-I in the

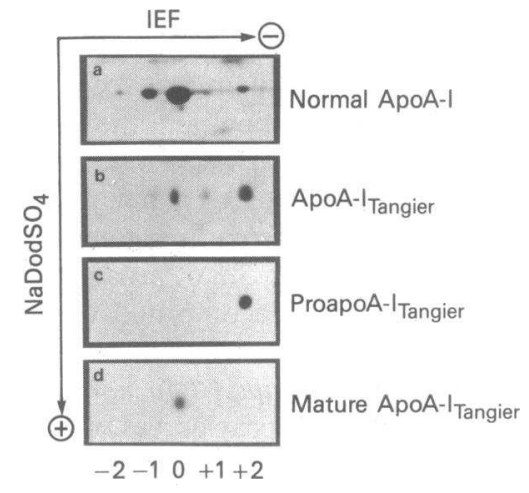

plasma gel containing the apoA-I isoforms is shown in the gel. Panels $(c)$ and $(d)$ are two-dimensional electrophoretograms of purified proapoA-I and mature apoA-I, respectively, from the plasma of the patient with Tangier disease.

Figure 1. Two-dimensional plasma gel electrophoretogram of apoA-I. Panel (a), apoA-I from a normal subject; panel $(b)$, apoA-I from a patient with Tangier disease. ProapoA-I is apoA-I isoforms +1 and +2 while mature apoA-I is isoforms $0,-1$, and -2 . Only the portion of the two-dimensional

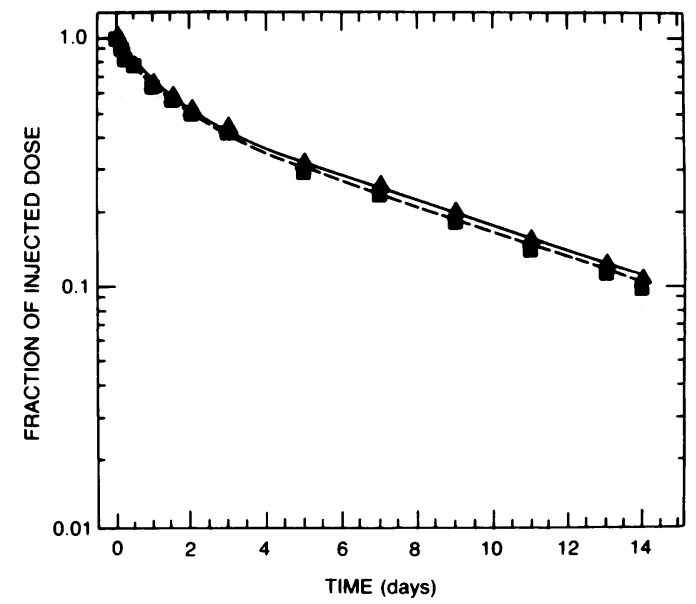

Figure 2. Plasma catabolic decay curves of radioiodinated normal proapoA-I ( $\square$ ) and mature apoA-I ( $\Delta$ ) injected into a normal subject. The curve depicts the total plasma decay curve without separation of the individual apoA-I isoforms. 


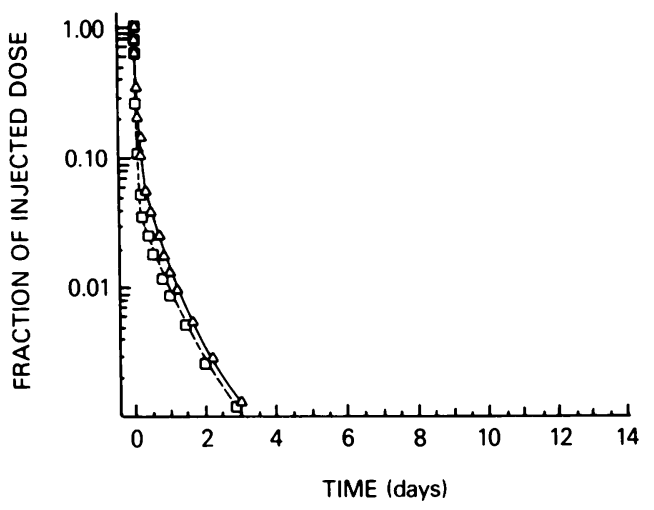

Figure 3. Plasma catabolic decay curves of proapoA-I ( $\square$ ) and mature apoA-I $(\Delta)$ isolated from a subject with Tangier disease, radioiodinated, and injected into a Tangier disease subject. The curves depict the total plasma decay curve without separation of the individual apoA-I isoforms.

Tangier disease subjects was the same as in normal subjects, and the synthesis rates of proapoA-I (i.e., newly secreted apoA-I) were similar to normal. Even though the fractional rate of conversion of proapoA-I to mature apoA-I was normal in these subjects, the absolute amount of proapoA-I converted was decreased. This was because $\sim 50 \%$ of the proapoA-I was directly catabolized from plasma before it was converted to mature apoA-I. Therefore, Tangier disease subjects had a normal apoA-I synthesis rate and normal fractional rate of conversion of proapoA-I to mature apoA-I. The decreased proapoA-I levels in these subjects are due to rapid catabolism of proapoA-I while the decreased levels of mature apoA-I are due to both a decreased conversion of proapoA-I to mature apoA-I in absolute terms and rapid catabolism of mature apoA-I.

Kinetic model of proapoA-I and mature apoA-I metabolism of Tangier disease subjects. Our current schematic model for apoA-I metabolism in normal and Tangier disease subjects is depicted in Fig. 5. The fractional conversion rates and the fractional catabolic rates of pro and mature apoA-I in both normal and Tangier disease subjects are obtained from the results of the above experiments. The width of the arrows is proportional to the absolute transport rates which were also obtained from the above experiments. The pool size values given at the bottom of the figure are calculated values from this

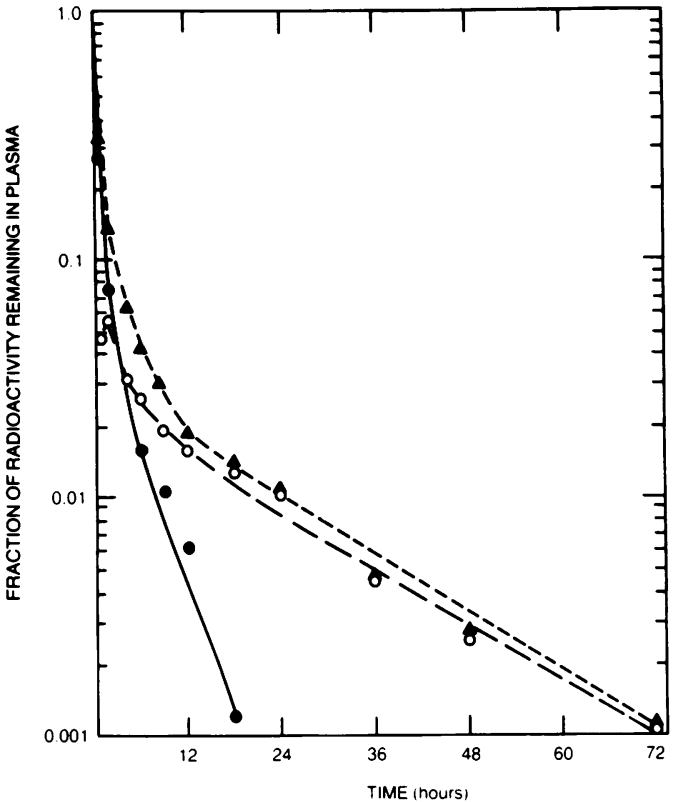

Figure 4. ApoA-I metabolic decay curves of proapoA-I and mature apoA-I in a Tangier disease subject. ProapoA-I and mature apoA-I were isolated from a Tangier disease subject, radioiodinated, and injected into a subject with Tangier disease. Timed plasma samples were collected following injection, plasma lipoproteins were isolated by ultracentrifugation, and the apoA-I isoforms separated by IEF gel electrophoresis. The curves for injected proapoA-I (๑), mature apoAI derived from the conversion of proapoA-I to mature apoA-I (O), and injected mature apoA-I ( $\Delta)$ are depicted on the figure.

model utilizing the given fraction rates of catabolism and conversion. All of the synthesis of apoA-I in both types of subjects is into the proapoA-I pool. In normal subjects, the fractional conversion rate is so much greater than the fractional catabolic or degradative rate for proapoA-I that virtually all of the proapoA-I is converted to mature apoA-I, and it is then slowly degraded. In Tangier disease subjects, the fractional catabolic rates of pro and mature apoA-I are markedly increased with the fractional catabolic rate now being approximately equal to the fractional conversion rate for proapoA-I. Therefore, $\sim 50 \%$ of the proapoA-I is directly degraded and $50 \%$ is converted to mature apoA-I followed by rapid degradation. The fractional catabolic and conversion rates that were utilized

Table II. Kinetics of Apolipoprotein A-I Metabolism in Normal and Tangier Disease Subjects

\begin{tabular}{|c|c|c|c|c|c|c|c|}
\hline \multirow[b]{2}{*}{ Subject } & \multicolumn{3}{|l|}{ ProapoA-I } & \multicolumn{3}{|l|}{ Mature ApoA-I } & \multirow[b]{2}{*}{ Conversion rate } \\
\hline & Concentration & $\mathbf{R T}^{*}$ & PR & Concentration & RT & PR & \\
\hline & $m g / d l$ & $d$ & $m g / k g-d$ & $m g / d l$ & $d$ & $m g / k g-d$ & $d^{-1}$ \\
\hline \multicolumn{8}{|c|}{ Normal } \\
\hline 1 & 5.5 & 0.19 & 11.6 & 133 & 5.2 & 10.2 & 3.1 \\
\hline 2 & 6.0 & 0.27 & 8.8 & 143 & 7.7 & 7.5 & 4.6 \\
\hline \multicolumn{8}{|c|}{ Tangier disease } \\
\hline 1 & 1.8 & 0.12 & 6.0 & 1.5 & 0.30 & 2.0 & 3.4 \\
\hline 2 & 1.1 & 0.06 & 7.3 & 0.5 & 0.13 & 1.5 & 3.7 \\
\hline
\end{tabular}

* RT, residence time; PR, production rate; conversion rate, fractional rate of conversion of proapoA-I to mature A-I. 


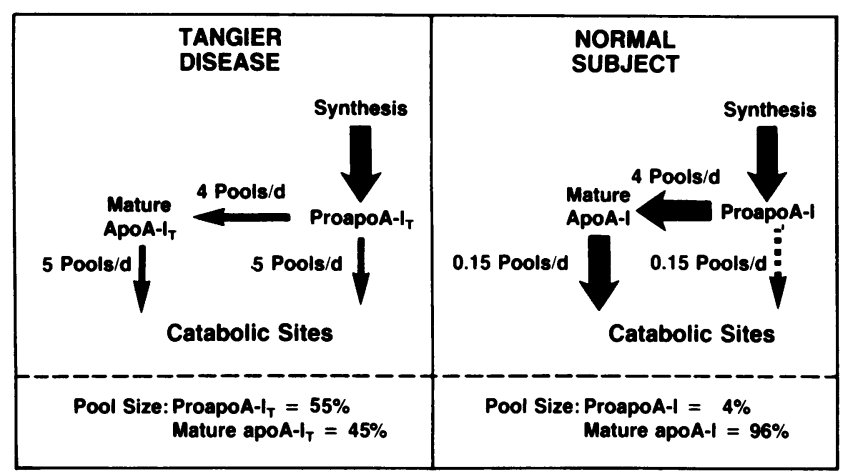

Figure 5. Scheme for the metabolism of apoA-I in normal subjects and Tangier disease subjects. The width of the arrows is proportional to the flux of material through that pathway, the fractional metabolic rates are derived from the above experimental results, and the pool size estimates at the bottom of the figure are calculated from the above model using the pathways and fractional metabolic rates given.

from the above studies predict very closely the independently determined densitometric gel scan distribution of plasma proapoA-I and mature apoA-I isoproteins in both normal and Tangier disease subjects.

\section{Discussion}

In normal subjects, apoA-I is secreted into plasma as proapoA-I, and while in the circulation is converted to mature apoA-I (5-9). This conversion is the result of cleavage of the amino terminal six amino acids of proapoA-I by a calcium requiring protease $(7,8)$. As we have previously demonstrated, the in vivo rate of conversion and the rate of catabolism of proapoA-I and mature apoA-I result in $\sim 95 \%$ of circulating apoA-I in normal subjects being the mature isoprotein while the remaining $5 \%$ is proapoA-I (9). In Tangier disease subjects, only 25 to $50 \%$ of the circulating apoA-I is the mature isoprotein while the remaining 50 to $75 \%$ is proapoA-I (10).

It has been proposed that apoA-I from subjects with Tangier disease is structurally abnormal (1), and that this structural abnormality results in rapid metabolism of apoA- $\mathrm{I}_{\text {Tangier }}$ (4) and a block in the conversion of proapoA-I $\mathrm{I}_{\text {Tangier }}$ to mature apoA-I I $_{\text {Tangier }}(5,10,11)$. In addition, it has been proposed that this structural abnormality results in an inability of proapoA$\mathbf{I}_{\text {Tangier }}$ to bind normally to lipoproteins (11). Our laboratory has recently determined the nucleotide, and derived amino acid sequence of preproapoA-I from a subject with Tangier disease (23). This subject had the characteristic Tangier disease distribution of apoA-I between the pro and mature isoproteins and rapid catabolism of apoA-I, and had an essentially normal preproapoA-I sequence. Therefore, we concluded that the abnormal apoA-I isoform distribution and rapid apoA-I catabolism in Tangier disease subjects is not secondary to a structural abnormality in the amino acid sequence of apoA-I.

It has also been proposed that the primary defect in Tangier disease may be an abnormality in the proapoA-I to mature apoA-I converting protease $(5,10,11)$. This abnormal protease would then lead to an accumulation of proapoA-I, and the proapoA-I would be catabolized very rapidly resulting in a very low plasma level of apoA-I and HDL cholesterol. In our investigations of the metabolism of proapoA-I and mature
apoA-I in normal subjects, proapoA-I was catabolized at the same rate as mature apoA-I (9). In addition, we and others have demonstrated with in vitro experiments, that normal and Tangier disease plasmas convert proapoA-I to mature apoA-I at the same rate $(8,24)$. Therefore, we concluded that the plasma proapoA-I to mature apoA-I converting protease activity is normal in Tangier disease subjects, that proapoA-I is not normally catabolized at a faster rate than mature apoA-I, and that the abnormal isoform distribution and rapid catabolism of apoA-I is not secondary to an abnormal converting protease activity in Tangier disease subjects $(8,9)$.

Our current investigations have elucidated the kinetic etiology of the abnormal plasma apoA-I isoform distribution in subjects with Tangier disease. This abnormal distribution is the result of a normal fractional rate of conversion of proapoA-I to mature apoA-I, and a rapid catabolism of both apoA-I isoproteins in subjects with Tangier disease. When proapoA-I is secreted into plasma, there are two possibilities for its catabolism. It may be directly catabolized from plasma or it may be converted to mature apoA-I and the mature apoA-I degraded. In normal subjects, the proapoA-I is rapidly converted to mature apoA-I with the slow catabolism of mature apoA-I resulting in a low plasma proapoA-I concentration compared to mature apoA-I. In subjects with Tangier disease, proapoA-I is secreted into plasma. The fractional rate of conversion to mature apoA-I is normal but the catabolic rate of the proapoA-I and mature apoA-I is increased 30 -fold. Since the rate of direct catabolism of proapoA-I and its rate of conversion to mature apoA-I are now nearly equal in these subjects, they catabolize $50 \%$ of their proapoA-I directly from plasma and convert $50 \%$ on to mature apoA-I. This results in a moderately decreased (by $\sim 50 \%$ ) proapoA-I concentrations in plasma from Tangier disease subjects as compared to normal plasma. It is not commonly appreciated that it is not the level of proapoA-I that is increased in Tangier disease but only the relative proportion of the proisoprotein of apoA-I compared to the mature apoA-I isoprotein.

In Tangier disease subjects, the plasma concentration of mature apoA-I is very low compared to normal plasma. In normal subjects $>95 \%$ of the proapoA-I is converted to mature apoA-I with slow catabolism of mature apoA-I; however, in Tangier disease only $50 \%$ of proapoA-I is converted to mature apoA-I. In addition to a decreased percentage of conversion of proapoA-I to mature apoA-I, the fractional catabolic rate of mature apoA-I is $\sim 30$ times greater in Tangier disease subjects than in normals. From these results it is apparent that the extremely low plasma concentrations of mature apoA-I in subjects with Tangier disease is a result of the rapid catabolism of both proapoA-I and mature apoA-I. Therefore, the kinetic explanation for the relative increase in proapoA-I in Tangier disease subjects is not an increase in proapoA-I secondary to a decreased rate of conversion of the proisoprotein to mature apoA-I, but a marked decrease in the mature isoprotein secondary to extremely rapid catabolism of apoA-I. It is of interest that the rapid rate of catabolism of proapoA-I and mature apoA-I in Tangier disease does not result in any marked alteration in the apoA-I synthesis rate.

What is unanswered, though, at the present time is the question of the biochemical explanation for the rapid catabolism of both proapoA-I and mature apoA-I. From the studies of the apoA-I sequence in subjects with Tangier disease, it is apparent that they synthesize and secrete a structurally normal 
proapoA-I (23). From the above and previous studies we conclude that Tangier disease subjects secrete proapoA-I at a normal rate, and that there is no defect in the fractional rate of conversion of proapoA-I to mature apoA-I in these subjects (8). There is a recent report of abnormal catabolism of HDL and apoA-I by monocyte-macrophages obtained from subjects with Tangier disease (25), but there have been no additional reports to confirm this finding in other kindreds. Therefore, it is still unknown as to why Tangier disease subjects rapidly catabolize apoA-I.

From the present studies we now have a kinetic explanation for the increase in the relative proportion of proapoA-I compared to mature apoA-I in Tangier disease subjects. This increase is not due to an increase in the concentration of proapoA-I but is secondary to a moderate decrease in the proisoprotein concentration and a very marked decrease in the concentration of the mature apoA-I isoprotein; both of these decreases in plasma concentrations being secondary to the rapid catabolism of both proapoA-I and mature apoA-I in Tangier disease subjects with a normal fractional rate of conversion of the pro-isoprotein to mature apoA-I. Additional studies are in progress to determine the biochemical etiology for the rapid catabolism of proapoA-I and mature apoA-I in subjects with Tangier disease.

\section{Acknowledgments}

We would like to thank Dr. Jimmy Light for supplying the human thoracic duct lymph, Mrs. Patti Riggs for dietary assistance in these studies, the nurses of the 8 East inpatient ward of the NIH Clinical Center for the nursing care of the study patients, Mrs. Imogene Surrey for preparing the manuscript, and especially the normal volunteers and Tangier disease patients for participating in these studies.

\section{References}

1. Herbert, P., G. Assmann, A. Gotto, and D. S. Fredrickson. 1983. Familial lipoprotein deficiency: abetalipoproteinemia, hypobetalipoproteinemia, and Tangier disease. Chapter 29. In The Metabolic Basis of Inherited Disease (5th ed.). J. B. Stanbury, J. B. Wyngaarden, D. S. Fredrickson, J. L. Goldstein, and M. S. Brown, editors. McGraw-Hill, New York. 607-621.

2. Schaefer, E. J., L. A. Zech, D. E. Schwartz, and H. B. Brewer, Jr. 1980. Coronary heart disease prevalence and other clinical features in familial high-density lipoprotein deficiency (Tangier Disease). Ann. Intern. Med. 93:261-266.

3. Schaefer, E. J., C. B. Blum, R. I. Levy, L. L. Jenkins, P. Alaupovic, D. M. Foster, and H. B. Brewer, Jr. 1978. Metabolism of highdensity lipoprotein apolipoproteins in Tangier Disease. N. Engl. J. Med. 299:905-910.

4. Schaefer, E. J., L. L. Kay, L. A. Zech, and H. B. Brewer, Jr. 1982. Tangier Disease: high density lipoprotein deficiency due to defective metabolism of an abnormal apolipoprotein A-I (apoA-I Tangier). $J$. Clin. Invest. 70:934-945.

5. Gordon, J. I., H. F. Sims, S. R. Lentz, C. Edelstein, A. M. Scanu, and A. W. Strauss. 1983. Proteolytic processing of human preproapolipoproteinA-I. A proposed defect in the conversion of proA-I to A-I in Tangier's disease. J. Biol. Chem. 258:4037-4044.

6. Brewer, H. B., Jr., T. Fairwell, L. Kay, M. Meng, R. Ronan, S. Law, and J. A. Light. 1983. Human plasma proapoA-I: isolation and amino-terminal sequence. Biochem. Biophys. Res. Commun. 113:626-632.
7. Edelstein, C., J. I. Gordon, K. Toscas, H. F. Sims, A. W. Strauss, and A. M. Scanu. 1983. In vitro conversion of proapoprotein A-I to apoprotein A-I. Partial characterization of an extracellular enzyme activity. J. Biol. Chem. 258:11430-11433.

8. Bojanovski, D., R. E. Gregg, and H. B. Brewer, Jr. 1984. Tangier disease: in vitro conversion of proapoA-I $\mathrm{I}_{\text {Tangier }}$ to mature apoA-I $\mathrm{I}_{\text {Tangier }}$. J. Biol. Chem. 259:6049-6051.

9. Bojanovski, D., R. E. Gregg, G. Ghiselli, E. J. Schaefer, J. A. Light, and H. B. Brewer, Jr. 1985. Human apolipoprotein A-I isoprotein metabolism: proapoA-I conversion to mature apoA-I. J. Lipid Res. 26:185-193.

10. Zannis, V. I., A. M. Lees, R. S. Lees, and J. L. Breslow. 1982. Abnormal apoprotein A-I isoprotein composition in patients with Tangier disease. J. Biol. Chem. 257:4978-4986.

11. Schmitz, G., G. Assmann, S. C. Rall, and R. W. Mahley. 1983. Tangier disease: defective recombination of a specific Tangier apolipoprotein A-I isoform (pro-apoA-I) with high density lipoproteins. Proc. Natl. Acad. Sci. USA. 80:6081-6085.

12. Fredrickson, D. S., P. H. Altrocchi, L. V. Avioli, D. S. Goodman, and H. C. Goodman. 1961. Tangier disease. Ann. Intern. Med. 55:1016-1031.

13. Havel, R. J., H. A. Eder, and J. H. Bragdon. 1955. The distribution and chemical composition of ultracentrifugally separated lipoproteins in human serum. J. Clin. Invest. 34:1345-1353.

14. Lowry, O. H., N. J. Rosebrough, A. L. Farr, and R. J. Randall. 1951. Protein measurement with the Folin phenol reagent. J. Biol. Chem. 193:265-275.

15. Schaefer, E. J., L. A. Zech, L. L. Jenkins, T. J. Bronzert, E. A. Rubalcaba, F. T. Lindgren, R. L. Aamodt, and H. B. Brewer, Jr. 1982. Human apolipoprotein A-I and A-II metabolism. J. Lipid Res. 23:850-862.

16. Brewer, H. B., Jr., T. Fairwell, A. LaRue, R. Ronan, A. House, and T. J. Bronzert. 1978. The amino acid sequence of human apoA-I, an apolipoprotein isolated from high density lipoproteins. Biochem. Biophys. Res. Commun. 80:623-630.

17. Brewer, H. B., Jr., T. Fairwell, M. Meng, L. Kay, and R. Ronan. 1983. Human proapoA-I $\mathrm{I}_{\text {Tangier }}$ : Isolation of proapoA-I $\mathrm{I}_{\text {Tangier }}$ and amino acid sequence of the propeptide. Biochem. Biophys. Res. Commun. 113:934-940.

18. MacFarlane, A. S. 1958. Efficient trace-labeling of proteins with iodine. Nature (Lond.). 182:53.

19. Gregg, R. E., L. A. Zech, E. J. Schaefer, and H. B. Brewer, Jr. 1984. Apolipoprotein E metabolism in normolipoproteinemic human subjects. J. Lipid Res. 25:1167-1176.

20. Sprecher, D. L., L. Taam, and H. B. Brewer, Jr. 1984. Human plasma apolipoproteins. Analysis by two-dimensional gel electrophoresis. Clin. Chem. 30:2084-2092.

21. Brewer, H. B., Jr., R. Ronan, M. Meng, and C. Bishop. 1986. Isolation and characterization of apolipoproteins A-I, A-II, and A-IV. In Methods in Enzymology. J. J. Athens and J. P. Seguert, editors. Vol. 128. Academic Press, Inc., New York. 223-246.

22. Anderson, D. W., E. J. Schaefer, T. J. Bronzert, F. T. Lindgren, T. Forte, T. E. Starzl, G. D. Niblack, L. A. Zech, and H. B. Brewer, Jr. 1981. Transport of apolipoprotein A-I and A-II by thoracic duct lymph. J. Clin. Invest. 67:857-866.

23. Law, S. W., and H. B. Brewer, Jr. 1985. Tangier Disease: the complete mRNA sequence encoding for preproapoA-I. J. Biol. Chem. 260:12810-12814.

24. Edelstein, C., J. I. Gordon, C. A. Vergani, A. L. Catapano, V. Pietrini, and A. M. Scanu. 1984. Comparative in vitro study of the proapolipoprotein A-I to apolipoprotein A-I converting activity between normal and Tangier plasma. J. Clin. Invest. 74:1098-1 103.

25. Schmitz, G., G. Assmann, H. Robenek, and B. Brennhausen. 1985. Tangier disease: A disorder of intracellular membrane traffic. Proc. Natl. Acad. Sci. USA. 82:6305-6309. 